\title{
Topological Fractional Pumping with Alkaline-Earth-Like Atoms in Synthetic Lattices
}

\author{
Luca Taddia, ${ }^{1}$ Eyal Cornfeld, ${ }^{2}$ Davide Rossini,${ }^{3,4}$ Leonardo Mazza, ${ }^{5}$ Eran Sela, ${ }^{2}$ and Rosario Fazio ${ }^{6,3}$ \\ ${ }^{1}$ Scuola Normale Superiore, I-56126 Pisa, Italy and CNR_Istituto Nazionale di Ottica, Sede Secondaria di Sesto Fiorentino, \\ I-50019 Sesto Fiorentino, Italy \\ ${ }^{2}$ Raymond and Beverly Sackler School of Physics and Astronomy, Tel-Aviv University, IL-69978 Tel Aviv, Israel \\ ${ }^{3}$ NEST, Scuola Normale Superiore and Istituto Nanoscienze-CNR, I-56126 Pisa, Italy \\ ${ }^{4}$ Dipartimento di Fisica, Università di Pisa and INFN, Largo Pontecorvo 3, I-56127 Pisa, Italy \\ ${ }^{5}$ Département de Physique, Ecole Normale Supérieure/PSL Research University, CNRS, 24 rue Lhomond, F-75005 Paris, France \\ ${ }^{6}$ ICTP, Strada Costiera 11, I-34151 Trieste, Italy
}

(Received 26 July 2016; revised manuscript received 13 March 2017; published 8 June 2017)

\begin{abstract}
Alkaline-earth(-like) atoms, trapped in optical lattices and in the presence of an external gauge field, can form insulating states at given fractional fillings. We will show that, by exploiting these properties, it is possible to realize a topological fractional pump. Our analysis is based on a many-body adiabatic expansion, on simulations with time-dependent matrix product states, and, for a specific form of atom-atom interaction, on an exactly solvable model of fractional pump. The numerical simulations allow us to consider a realistic setup amenable of an experimental realization. As a further consequence, the measure of the center-of-mass shift of the atomic cloud would constitute the first measurement of a many-body Chern number in a cold-atom experiment.
\end{abstract}

DOI: 10.1103/PhysRevLett.118.230402

Introduction.-Since the invention of the Archimedean screw, it has been known that matter and energy can be transported, or pumped, without imposing any external bias, but with a periodic modulation of some system parameters. Investigations of pumping in quantum systems encompass a wide range of phenomena and applications (see, e.g., Refs. [1,2]). In the adiabatic limit, quantum pumping is of geometric nature, being related to the Berry phase (or its generalizations) accumulated during the cycle [3]; in some cases, the phase has a further topological nature and displays quantization properties. This is what Thouless first considered [4], showing that in certain onedimensional insulators the pumped charge is quantized; the experimental demonstration had to wait for three decades till its recent realization with cold atoms $[5,6]$.

Interest in states of matter supporting forms of fractionalization (e.g., interacting topological systems [7-12]) grew enormously in recent years. An appealing exploration avenue is the search for signatures in measurable quantities, such as the quantization to rational values of the conductance characterizing the fractional quantum Hall effect (QHE) [13]. With this motivation, we here investigate the probe of phases of matter supporting the topological pump of a fractional charge.

In this Letter, we focus on cold atoms, where, despite numerous experimental results for free systems [14-18], evidence for fractional topological properties is still missing. We discuss a realistic fractional pumping experiment for interacting alkaline-earth(-like) atoms trapped in onedimensional optical lattices and subjected to an external gauge field. The measurement of the center-of-mass motion of the gas gives access to the pumped charge and thus to the many-body Chern number of the ground states $[19,20]$. This is different from measuring the Chern number of the energy bands of free systems, as reflected by the quantization to fractional values of the pumped charge (or better mass, since atoms are neutral).

Our work is motivated by several previous theoretical studies. The connection between Chern numbers and center-of-mass motion of the cloud was considered in Refs. [21-24]. Using a static analysis of the Hamiltonian spectral flow, some aspects of fractional pumping were discussed for specific bosonic models [25] and fermionic ladders [26], although in the latter case quantization is not expected due to gapless edge modes. Topological pumping was addressed in interacting bosons on a lattice with inversion symmetry breaking $[27,28]$ and in photonic systems with nonlinear resonators arrays [29].

Our proposal for a fractional topological pump is already realizable thanks to the progress of experiments with alkalineearth(-like) gases [5,30-39], encompassing the observations of interaction effects [34] and the engineering of synthetic gauge fields [35]. This calls for a detailed dynamical analysis of pumping in this system: we accomplish this task using an adiabatic expansion and time-dependent many-body algorithms based on matrix product states. We address finite-size and nonadiabatic corrections, the role of the trap, and the detection protocol $[5,40]$. By elucidating the relation between the pump and the topology of many-body states, we also clarify their relation with the fractional QHE $[13,41]$.

Model.-We consider a one-dimensional gas of $N$ alkaline-earth(-like) fermions with $2 \mathcal{I}+1$ nuclear spin states [31,34] coupled through Raman beams [35-37,42] and trapped in an optical lattice (see Fig. 1 for $\mathcal{I}=1$ ). 


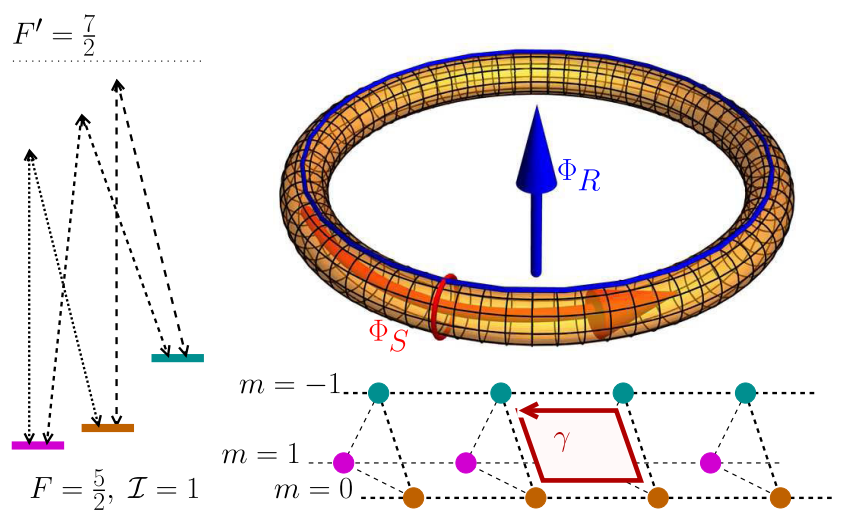

FIG. 1. Left: Three contiguous nuclear spin states of ${ }^{173} \mathrm{Yb}$ $(F=5 / 2)$ are coupled through independent Raman couplings which adiabatically eliminate long-lived excited states $\left({ }^{3} P_{1}\right.$, $F^{\prime}=7 / 2$ ). Energies are not in scale. By coupling only a subset of the nuclear spin states, an effective spin $\mathcal{I}=1$ is obtained. Right: The system geometry with periodic boundaries is a thin torus with a long real dimension and a length-three synthetic one. Two fluxes, $\Phi_{R}$ and $\Phi_{S}$, pierce the noncontractible circles. A flux $\gamma$ trades every synthetic plaquette.

The Hamiltonian, in the single-band approximation, reads $(\hbar=1)$

$$
\begin{aligned}
\hat{H}= & \sum_{j, m}\left[-t \hat{c}_{j, m}^{\dagger} \hat{c}_{j+1, m}+\Omega e^{-i \gamma j} \hat{c}_{j, m}^{\dagger} \hat{c}_{j, m+1}+\text { H.c. }\right] \\
& +\sum_{i, j, m, m^{\prime}} U_{i, j}^{m, m^{\prime}} \hat{n}_{i, m} \hat{n}_{j, m^{\prime}}+w_{0} \sum_{j, m}\left(j-j_{0}\right)^{2} \hat{n}_{j, m} .
\end{aligned}
$$

The operator $\hat{c}_{j, m}^{(\dagger)}$ annihilates (creates) a fermion of nuclear spin $m=-\mathcal{I}, \ldots, \mathcal{I}$ at site $j=1, \ldots, L$ (here, $\hat{n}_{j, m}=$ $\hat{c}_{j, m}^{\dagger} \hat{c}_{j, m}$ and $\hat{n}_{j}=\sum_{m} \hat{n}_{j, m}$ ). The first term in the rhs represents the hopping along the optical lattice, with amplitude $t$. The second term accounts for spin flips induced by Raman beams (Fig. 1); we take $\Omega$ independent of $m$ since experimental schemes exist which allow for setting them to the approximatively same value [43]. The term corresponding to $\hat{c}_{j, \mathcal{I}}^{\dagger} \hat{c}_{j, \mathcal{I}+1}$ should be understood as $\hat{c}_{j, \mathcal{I}}^{\dagger} \hat{c}_{j,-\mathcal{I}}$, requiring a similarly engineered Raman transition [43]. As experimentally realized [35], the angle between Raman beams induces a site-dependent complex amplitude $e^{-i \gamma j}$, with $\gamma$ mimicking a static gauge potential. Interactions are typically short-ranged and $\mathrm{SU}(2 \mathcal{I}+1)$ invariant $[32,44]$; this leads to the choice $U_{i, j}^{m, m^{\prime}}=(U / 2)\left[1-\delta_{m, m^{\prime}}\right] \delta_{i, j}+V \delta_{j, i+1}$ with onsite and nearest-neighbor couplings $U$ and $V$, respectively. The experimentally relevant case $V=0$ is discussed in the final part of the Letter. The last term in Eq. (1) is the harmonic confinement of strength $w_{0}$, centered around $j_{0}=(L+1) / 2$. We will study a lattice of $L$ sites both with periodic and open boundary conditions; while the former case allows for an elegant analytical approach, the latter one is experimentally relevant.
The Hamiltonian Eq. (1) can be seen as a spinlessfermion model on a $L \times(2 \mathcal{I}+1)$ cylinder (torus) for open (periodic) boundaries [45] pierced by a flux per plaquette $\gamma$ [42]. The spin index $m$ acquires the meaning of a lattice label in the synthetic direction (Fig. 1); in this Letter, the system always has periodic boundary conditions in the synthetic dimension. At fractional values of filling [46], $\nu \equiv 2 \pi N /[(2 \mathcal{I}+1) L \gamma]=p / q$, a gapped phase may form [36]; here, the ground states display both density and spin order. The charge order is visible, for example, in the lefthand panel of Fig. 3. Because of the presence of a spiral-like ordering in the (nuclear-spin) magnetization, these phases have been termed magnetic crystals [36].

In order to realize a pump, the amplitudes $t$ and $\Omega$ in Eq. (1) must vary in time [37]; i.e., $t \rightarrow t(\tau)=t e^{i \Phi_{R}(\tau) / L}$ and $\Omega \rightarrow \Omega(\tau)=\Omega e^{i \Phi_{S}(\tau) /(2 \mathcal{I}+1)}$. The phases $\Phi_{R, S}(\tau)$ represent time-dependent fluxes piercing, respectively, real and synthetic circles (Fig. 1). The crystals realize a fractional topological pump by adiabatically varying them from 0 to $2 \pi$ in a period $T$. This scheme parallels Laughlin's argument for QHE [47].

Topological fractional pump.-We first consider periodic boundaries and no trap. We characterize the pump by performing a next-to-adiabatic expansion [48] of the Schrödinger equation $T^{-1} i \partial_{s}|\Psi(s)\rangle=\hat{H}(s)|\Psi(s)\rangle$ with rescaled time $s=\tau / T$ and assuming a slow periodic variation of the phases $\Phi_{R, S}(\tau)$. The magnetic crystals at filling $\nu=p / q$ are $q$-fold degenerate on a torus. For any instantaneous energy level $E_{n}(s)$, we introduce the multiplet of eigenvectors $\left|n^{h}(s)\right\rangle$. Thus, $\left|0^{h}(s=0)\right\rangle$, with $h=1, \ldots, q$, represents one of the ground states in which the system is initialized.

We focus on pumping along the real direction, with $\Phi_{S}(\tau)=2 \pi \tau / T$ and $\Phi_{R}$ kept constant in time (one can similarly analyze a possible pump in the synthetic dimension [49]). The atomic mass transferred in one period when the system is initialized in $\left|0^{h}(s=0)\right\rangle$ is $Q_{j}^{(h)}(T)$, where $Q_{j}^{(h)}(\tau)=T \sum_{m} \int_{0}^{\tau / T} d s\left\langle\hat{J}_{j, m}^{(R)}\left(\Phi_{R}\right)\right\rangle^{(h)}$ and the current operator is $\hat{J}_{j, m}^{(R)}\left(\Phi_{R}\right)=i t e^{i \Phi_{R} / L} \hat{c}_{j, m}^{\dagger} \hat{c}_{j+1, m}+$ H.c. We use the notation $Q^{(h)}$ for values averaged over different sites, dropping $T$ when referring to one cycle. This expression can be expanded in powers of $T^{-1}$ using the next-toadiabatic expansion for $\left|\Psi^{h}(s)\right\rangle$ mentioned above (see also Refs. [50,51]). The zeroth-order term accounts for the ground-state persistent current, and vanishes when $\Phi_{R}=0$. The quantized pumped mass $Q_{P}$ follows from the firstorder term; higher-order ones account for nonadiabatic corrections. Further details are given in the Supplemental Material [49].

Since the pumped mass $Q_{P}$ is independent of the initial eigenstate, it is convenient to average over the $q$ possible initial states, $Q_{P}=q^{-1} \sum_{h} Q^{(h)}$, obtaining [49]

$$
Q_{P}=i \int_{0}^{2 \pi} d \Phi_{S} \frac{1}{q} \sum_{h}\left[\Omega_{\mathrm{WZ}}\right]^{h h} .
$$


Because of ground-space degeneracy, $Q_{P}$ is related to the Wilczek-Zee (WZ) [52] curvature matrix $\left[\Omega_{\mathrm{WZ}}\right]^{h^{\prime} h^{\prime \prime}}=$ $i\left[\left\langle\partial_{\Phi_{S}} 0^{h^{\prime}} \mid \partial_{\Phi_{R}} 0^{h^{\prime \prime}}\right\rangle+\sum_{h}\left\langle\partial_{\Phi_{S}} 0^{h^{\prime}} \mid 0^{h}\right\rangle\left\langle 0^{h} \mid \partial_{\Phi_{R}} 0^{h^{\prime \prime}}\right\rangle\right]+$ H.c. By averaging over the constant flux $\Phi_{R}$, we obtain

$$
\left\langle Q_{P}\right\rangle_{\Phi_{R}}=\frac{1}{q} \oiint \frac{d^{2} \Phi}{2 \pi} \sum_{h}\left[\Omega_{\mathrm{WZ}}\right]^{h h}=\frac{\mathcal{C}_{1}}{q} .
$$

The closed surface integral over the trace of the curvature $\mathcal{C}_{1}$ is the integer topological invariant known as the nonAbelian first Chern number [53]. Equation (3) is crucial for our proposal: it shows that a system whose ground state is degenerate can pump a charge that is topological, fractional, and related to the many-body Chern number. In the following, we demonstrate that model Eq. (1) features such a property when its parameters are such that the ground state is a magnetic crystal.

A first glimpse of topological pumping with magnetic crystals is provided by the following exactly solvable model. We consider a hard-core interaction of range $\xi$ in the real dimension, $U_{i, j}^{m, m^{\prime}} \rightarrow \infty$ for $|i-j| \leq \xi$ and zero otherwise. We can eliminate the $\xi$ empty rungs to the right of the $N-1$ particles along the real dimension, and write an effective model on a system of reduced length, $L^{\prime}=L-(N-1) \xi$ $[49,54,55]$ (for open boundary conditions). Hard-core particles hop in the reduced lattice similarly to before: this part of the Hamiltonian is formally unchanged [49]. The spin flip is also formally unchanged, but the site-dependent phase $\gamma j$ is replaced by $\gamma j+\gamma \xi \sum_{j^{\prime}=1}^{j-1} \hat{n}_{j^{\prime}}$, thus introducing a nonlocal term. When the flux satisfies $\gamma \xi=2 \pi a$, with $a \in \mathbb{N}$, the model turns local again. Through this flux-attachment transformation [56], we obtain a model characterized by hard-core on-site interactions with modified filling $\nu^{\prime}=\nu L / L^{\prime}$. Thus, when $L^{\prime} / L=q(q \in \mathbb{N})$, the mapping relates the fractional filling $\nu^{\prime}=p / q$ to the integer filling $\nu=p$. In these cases, we can relate the topological integral $\left\langle Q_{P}\right\rangle_{\Phi_{R}}$ in Eq. (3) to $\left\langle Q_{P}^{\prime}\right\rangle_{\Phi_{R}}$ (the pumping in the effective model) by observing that $\Phi_{R} / L$ in the time-dependent Hamiltonian equals $\Phi_{R} q / L^{\prime}$, thus obtaining: $\left\langle Q_{P}\right\rangle_{\Phi_{R}}=\left\langle Q_{P}^{\prime}\right\rangle_{\Phi_{R}} / q$ [49]. To compute $\left\langle Q_{P}^{\prime}\right\rangle_{\Phi_{R}}$, we observe that for zero on-site interaction the system is in a gapped phase characterized by $\left\langle Q_{P}^{\prime}\right\rangle_{\Phi_{R}}=p$. Since we expect that repulsive interactions stabilize the gap without encountering phase transitions, $\left\langle Q_{P}^{\prime}\right\rangle_{\Phi_{R}}=p$ also for our effective model [49]. Concluding, $\left\langle Q_{P}\right\rangle_{\Phi_{R}}=p / q$.

The corrections to pumping due to finite-size and nonadiabatic effects are estimated as [57]

$$
Q_{P}=\mathcal{C}_{1} / q+O\left(L^{-1}\right)+O\left((T \Delta)^{-1} f(T \Delta)\right),
$$

where $\Delta$ is the time-averaged energy gap. The first correction stems from the fact that, in general, changing a twisted boundary condition into an open one introduces a difference scaling as $1 / L$ (averaging over $\Phi_{R}$ cancels the error even at finite size). Nonadiabatic corrections descend from explicit inspection, and $f(x) \sim A+B \cos (x+\phi)(A$, $B, \phi$ are computable constants) [49].

Numerical simulations. - The model presented above is not experimentally relevant at present. We thus simulate Eq. (1) for $\mathcal{I}=1$ and $\gamma=\pi / 3$, focusing on the fractions $\nu=1 / 2$ (with no counterpart in $2 \mathrm{D}$ Hall physics) and $\nu=1 / 3$. Nearest-neighbor interactions are needed to stabilize both crystals [36]. Simulations are based on a timedependent matrix-product-state approach: the evolution operator is decomposed using a fourth-order Trotter approximation with time steps between $10^{-3} t^{-1}$ and $10^{-2} t^{-1}$, keeping a bond link up to 200 [58]. Open boundaries and pumping in the real dimension $\left(\Phi_{R}=0\right)$, relevant for experiments, are considered.

The left-hand panel of Fig. 2 shows, at fixed $T$, the spaceresolved pumped charge $Q_{j}^{(h)}(\tau)$ for a specific crystal labeled by $h$. Since it is inhomogeneous, it displays a dependence on $j$, with periodicity $\ell=4$ for $\nu=1 / 2$ and $\ell=6$ for $\nu=1 / 3(\ell$ is the space periodicity of the density profile; here, $\ell=n^{-1}$, where $n=N / L$ is the particle density). As the average over $\ell$ sites $Q_{\text {av }}$ does not depend on $h$ [while $Q_{j}^{(h)}(\tau)$ does, due to the hard boundaries], we can drop the apex $(h)$ and quantization is restored after performing a spatial average over $\ell$ sites. In the right-hand panels, we show that the $T$ dependence of this averaged value $Q_{\mathrm{av}}(\tau=T)$ oscillates around the quantized values, with a vanishing amplitude in the adiabatic limit (the charge transferred in a fraction of the pumping period is also quantized [59]).

The quantization of $Q_{\mathrm{av}}$ can also be observed by looking at the dynamics of magnetic crystals. In the left-hand panel of Fig. 3, we show the time evolution of density profiles at $\nu=1 / 3$ : for large $T$, the particles moved by two sites $[25,37]$. To understand this, we consider the eigenvalues of
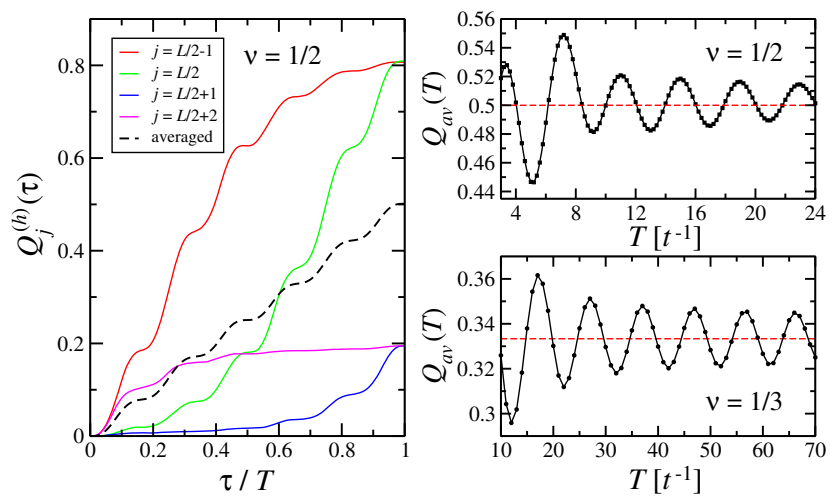

FIG. 2. Pumped charge after one period for a system with hardwall boundaries, $L=48, \gamma=\pi / 3, U / t=\infty, V / t=10 ; \Omega / t=$ 1 for $\nu=1 / 2$ and $\Omega / t=0.25$ for $\nu=1 / 3$. Left: Space-resolved charge $Q_{j}^{(h)}(\tau)$ for $\nu=1 / 2$ and $T=24$. Right: Pumped charge $Q_{\text {av }}(T)$ as a function of period $T$ for $\nu=1 / 2$ (top) and $\nu=1 / 3$ (bottom). Dashed lines show quantized values. The pumped charge is independent of the initial eigenstate. 

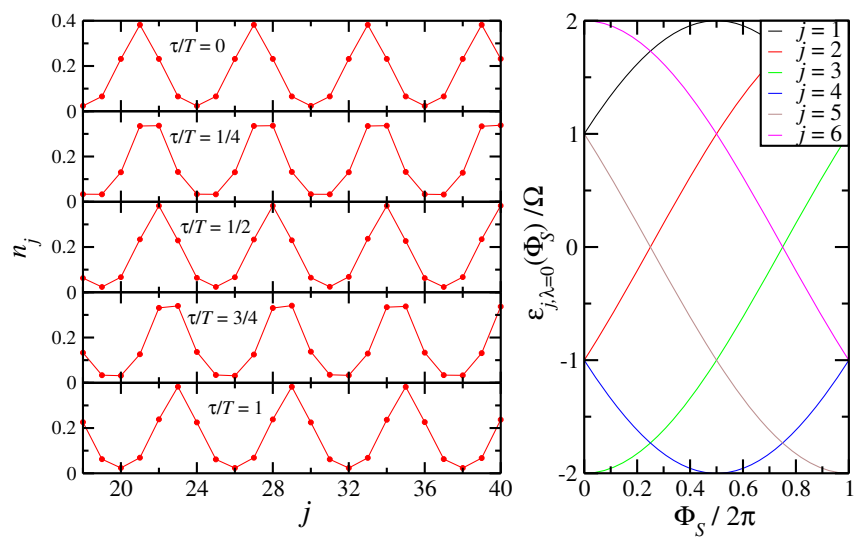

FIG. 3. Left: Time evolution of the density profile at $\nu=1 / 3$. The parameters are specified in the caption of Fig. $2 ; T=100 t^{-1}$. For such a value of $T$, the system is essentially in the instantaneous ground state at all times. Right: Flux dependence of $\epsilon_{j, \lambda=0}\left(\Phi_{S}\right)$.

the spin-flip term [the one proportional to $\Omega$ in Eq. (1)], $\epsilon_{j, \lambda}\left(\Phi_{S}\right)=\cos \left[\left(2 \pi \lambda-\Phi_{S}\right) /(2 \mathcal{I}+1)+\gamma j\right]$, which are labeled by $\lambda=0, \ldots, 2 \mathcal{I}$. They correspond to momentum states along the synthetic dimension. In the right-hand panel we plot their adiabatic time evolution for $j=1, \ldots, 6$ and $\lambda=0$. Because of hopping, the crossings turn into avoided crossings which can be adiabatically followed for large enough $T$. A particle initially sitting at $j=3$ is transported to $j=4$ at $\tau=T / 2$ (or $\Phi_{S}=\pi$ ) and then to $j=5$ at $\tau=T\left(\right.$ or $\left.\Phi_{S}=2 \pi\right)$. From the definition of $\epsilon_{j, \lambda}$ we infer that, in a pump period, particles are adiabatically transported along $\Delta j=2 \pi /[(2 \mathcal{I}+1) \gamma]$ sites. The net pumped mass is given by the product $n \Delta j \equiv \nu$, which is fractional, and agrees with our numerics.

It is intriguing that the pumped charge is related to a manybody Chern number, the topological invariant that characterizes the wave functions of the fractional QHE by twisting the boundary conditions. Since magnetic crystals are related to the QHE in the thin-torus limit, where the second dimension is the shortest length scale, this shows that in this limit the topological invariant persists. However, these states do not display topological order, as reflected by the existence of crystals at all fillings $\nu=p / q$, whereas in two dimensions the fractional QHE states only appear for odd $q$. Thus, fractional pumping does not require the existence of a counterpart gapped state in the two-dimensional version of the QHE and appears in all fractional gapped states of the anisotropic limit.

Experimental detection.-In order to ascertain the possibility of measuring the fractional pump, we consider the role of a trapping potential and analyze measurable quantities that carry information about pumping. We switch to $\gamma=2 \pi / 3$, which allows the appearance of magnetic crystals at $\nu=1 / 2$ in the relevant case of no nearest-neighbor interaction, $V=0$.

The pumped charge can be experimentally estimated through the center-of-mass displacement of the cloud
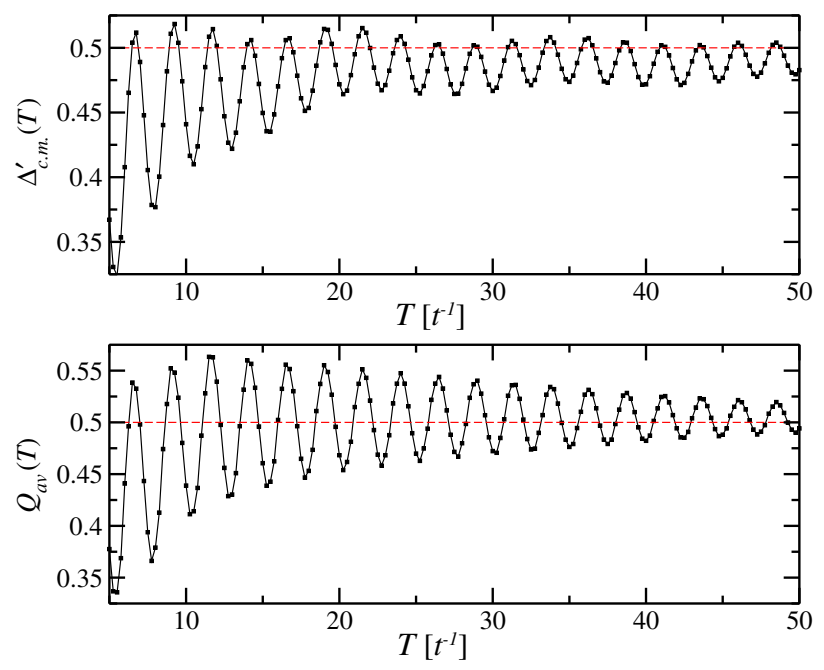

FIG. 4. Center-of-mass displacement of the cloud $\Delta_{\text {c.m. }}(T) / \ell$ (top) and pumped charge $Q_{\mathrm{av}}(T)$ (bottom) versus $T$ for a magnetic crystal with $\nu=1 / 2$ in a harmonic potential. Dashed lines show quantized values. In order to observe the oscillations due to nonadiabatic corrections, a time resolution $\sim t^{-1}$ is needed. The figure indicates that the small metallic wings at the crystal edges, due to the harmonic potential, do not significantly affect the quantization. Parameters: $\gamma=2 \pi / 3, U / t=\infty, \Omega / t=1$, $w_{0} / t=0.01, L=48, N=10$.

$\Delta_{\text {c.m. }}(T)$ [40]. At low fillings, like in Fig. 4, the system is almost entirely in a magnetic crystal and the motion of the whole cloud can be directly related to pumping. In the top panel, we plot $\Delta_{\text {c.m. }}^{\prime}(T)=\Delta_{\text {c.m. }}(T) / \ell$ [40]. We observe a good quantization of $\Delta_{\text {c.m. }}^{\prime}$, and an excellent comparison with the pumped charge $Q_{\mathrm{av}}(T)$ (bottom panel), apart from the expected nonadiabatic corrections. The harmonic trap helps the pumping, because the soft boundaries act as source and drain leads.

Ultracold ${ }^{173} \mathrm{Yb}$ gases with $\mathcal{I}=1$ have already been realized [34,35], and realistic schemes exist for multiphoton coupling between $m= \pm 1$ states $[37,43] . \Phi_{S}$ is a global phase associated to the spin-flip Raman beams, and can be manipulated in time [37]. $\Phi_{R}$ is instead irrelevant. Measurements of center-of-mass displacements can be performed [5], and executing several pump cycles can make the effect more detectable. Real-space currents can be detected by asymmetries in the spin-resolved momentum distribution function measured with time-of-flight imaging [35,38]. The largest value $T=50 t^{-1}$ in Fig. 4 corresponds to $80 \mathrm{~ms}$, taking $t \sim 2 \pi \times 100 \mathrm{~Hz}$.

In conclusion, we comment on two additional sources of imperfect quantization: the presence of multiple copies of the system and the effect of finite temperature. A microscope with single-site addressing resolution would allow the possibility of analyzing only the tubes where the density-flux commensurability requirement is matched. Alternatively, following the approach of Ref. [6], an optical superlattice can be used to adiabatically turn a Mott insulator with one particle every two 
sites into a magnetic crystal. We performed numerical simulations to analyze an adapted version of the adiabatic preparation to our case; the results, detailed in the last section of Ref. [49], show that this approach can be very fruitful. Following Ref. [6], almost all tubes are carved out of the Mott insulator, and thus produce the fractional pumping discussed thus far. The presence of a thin surrounding superfluid shell, introducing a spurious signal, was tamed in Ref. [6] and thus is predicted to be inessential also here. Regarding temperature, it should be lower than the gap (see Ref. [4] for the integer Thouless pump); for $\nu=1 / 2$, the gap is comparable to $t$ [36].

If experimentally realized, this measurement would constitute a pioneering direct observation of a many-body Chern number.

We thank S. Barbarino, M. Burrello, M. Calvanese Strinati, G. Cappellini, J. Catani, M. Dalmonte, L. Livi, M. Mancini, G. Pagano, C. Sias, and especially L. Fallani for valuable discussions, and S. Sinigardi for technical support. We are grateful to M. Aidelsburger for suggestions on the adiabatic state preparation scheme. We acknowledge INFN-CNAF for providing us computational resources and support, and D. Cesini in particular; we also acknowledge the CINECA award under the ISCRA initiative, for the availability of highperformance computing resources and support. We acknowledge financial support of EU-IP-SIQS (L.T., D. R., and R.F.), EU-IP-QUIC (R.F.), SNS 2014 Projects (R.F.), QSYNC (R. F.), LabEX ENS-ICFP: ANR-10-LABX-0010/ ANR-10-IDEX-0001-02 PSL* (L. M.), ISF Grant No. 1243/ 13, and the Marie Curie CIG Grant No. 618188 (E. S.).

Note added.-Recently, we became aware of a related work by Zeng, Zhu, and Sheng [60] for the fractional pumping in interacting bosonic systems.

[1] J. P. Pekola, J. J. Vartiainen, M. Möttönen, O.-P. Saira, M. Meschke, and D. V. Averin, Nat. Phys. 4, 120 (2008).

[2] J. E. Avron, D. Osadchy, and R. Seiler, Phys. Today 56, No. 838 (2003).

[3] D. Xiao, M.-C. Chang, and Q. Niu, Rev. Mod. Phys. 82, 1959 (2010).

[4] D. J. Thouless, Phys. Rev. B 27, 6083 (1983).

[5] S. Nakajima, T. Tomita, S. Taie, T. Ichinose, H. Ozawa, L. Wang, M. Troyer, and Y. Takahashi, Nat. Phys. 12, 296 (2016)

[6] M. Lohse, C. Schweizer, O. Zilberberg, M. Aidelsburger, and I. Bloch, Nat. Phys. 12, 350 (2016).

[7] C. Nayak, S. H. Simon, A. Stern, M. Freedman, and S. Das Sarma, Rev. Mod. Phys. 80, 1083 (2008).

[8] M. Z. Hasan and C. L. Kane, Rev. Mod. Phys. 82, 3045 (2010).

[9] X.-L. Qi and S.-C. Zhang, Rev. Mod. Phys. 83, 1057 (2011).

[10] L. Fidkowski and A. Kitaev, Phys. Rev. B 81, 134509 (2010).
[11] A. M. Turner, F. Pollmann, and E. Berg, Phys. Rev. B 83, 075102 (2011).

[12] C.-K. Chiu, J. C. Y. Teo, A. P. Schnyder, and S. Ryu, Rev. Mod. Phys. 88, 035005 (2016).

[13] The Quantum Hall Effect, edited by R. E. Prange and S. M. Girvin (Springer-Verlag, New York, 1990).

[14] L. Duca, T. Li, M. Reitter, I. Bloch, M. Schleier-Smith, and U. Schneider, Science 347, 288 (2015).

[15] T. Li, L. Duca, M. Reitter, F. Grusdt, E. Demler, M. Endres, M. Schleier-Smith, I. Bloch, and U. Schneider, Science 352, 1094 (2016).

[16] H.-I. Lu, M. Schemmer, L. M. Aycock, D. Genkina, S. Sugawa, and I. B. Spielman, Phys. Rev. Lett. 116, 200402 (2016).

[17] M. Aidelsburger, M. Lohse, C. Schweizer, M. Atala, J. T. Barreiro, S. Nascimbène, N. R. Cooper, I. Bloch, and N. Goldman, Nat. Phys. 11, 162 (2015).

[18] N. Fläschner, B. S. Rem, M. Tarnowski, D. Vogel, D.-S. Lühmann, K. Sengstock, and C. Weitenberg, Science 352, 1091 (2016).

[19] R. Tao and F. D. M. Haldane, Phys. Rev. B 33, 3844 (1986).

[20] T. Ozawa, H. M. Price, and I. Carusotto, Phys. Rev. B 93, 195201 (2016).

[21] H. M. Price and N. R. Cooper, Phys. Rev. A 85, 033620 (2012).

[22] A. Dauphin and N. Goldman, Phys. Rev. Lett. 111, 135302 (2013).

[23] T. Ozawa and I. Carusotto, Phys. Rev. Lett. 112, 133902 (2014).

[24] H. M. Price, O. Zilberberg, T. Ozawa, I. Carusotto, and N. Goldman, Phys. Rev. B 93, 245113 (2016).

[25] F. Grusdt and M. Höning, Phys. Rev. A 90, 053623 (2014).

[26] T.-S. Zeng, C. Wang, and H. Zhai, Phys. Rev. Lett. 115, 095302 (2015).

[27] E. G. Dalla Torre, E. Berg, and E. Altman, Phys. Rev. Lett. 97, 260401 (2006).

[28] D. Rossini, M. Gibertini, V. Giovannetti, and R. Fazio, Phys. Rev. B 87, 085131 (2013).

[29] J. Tangpanitanon, V. M. Bastidas, S. Al-Assam, P. Roushan, D. Jaksch, and D. G. Angelakis, Phys. Rev. Lett. 117, 213603 (2016).

[30] T. Fukuhara, Y. Takasu, M. Kumakura, and Y. Takahashi, Phys. Rev. Lett. 98, 030401 (2007).

[31] A. V. Gorshkov, M. Hermele, V. Gurarie, C. Xu, P. S. Julienne, J. Ye, P. Zoller, E. Demler, M. D. Lukin, and A. M. Rey, Nat. Phys. 6, 289 (2010).

[32] M. A. Cazalilla and A. M. Rey, Rep. Prog. Phys. 77, 124401 (2014).

[33] X. Zhang, M. Bishof, S. L. Bromley, C. V. Kraus, M. S. Safronova, P. Zoller, A. M. Rey, and J. Ye, Science 345, 1467 (2014).

[34] G. Pagano, M. Mancini, G. Cappellini, P. Lombardi, F. Schäfer, H. Hu, X.-J. Liu, J. Catani, C. Sias, M. Inguscio, and L. Fallani, Nat. Phys. 10, 198 (2014).

[35] M. Mancini, G. Pagano, G. Cappellini, L. Livi, M. Rider, J. Catani, C. Sias, P. Zoller, M. Inguscio, M. Dalmonte, and L. Fallani, Science 349, 1510 (2015).

[36] S. Barbarino, L. Taddia, D. Rossini, L. Mazza, and R. Fazio, Nat. Commun. 6, 8134 (2015). 
[37] N. R. Cooper and A. M. Rey, Phys. Rev. A 92, 021401(R) (2015).

[38] S. Barbarino, L. Taddia, D. Rossini, L. Mazza, and R. Fazio, New J. Phys. 18, 035010 (2016).

[39] C. Hofrichter, L. Riegger, F. Scazza, M. Höfer, D. R. Fernandes, I. Bloch, and S. Fölling, Phys. Rev. X 6, 021030 (2016).

[40] L. Wang, M. Troyer, and X. Dai, Phys. Rev. Lett. 111, 026802 (2013).

[41] E. J. Bergholtz and A. Karlhede, Phys. Rev. B 77, 155308 (2008).

[42] A. Celi, P. Massignan, J. Ruseckas, N. Goldman, I. B. Spielman, G. Juzeliūnas, and M. Lewenstein, Phys. Rev. Lett. 112, 043001 (2014).

[43] Referring to the case $\mathcal{I}=1$, it is possible to resolve the different spin-flip transitions by applying a spin-dependent light shift (mimicking a nonlinear Zeeman shift) and putting proper sidebands on the frequency of the two Raman beams in order to address and control each $\Omega$ by adjusting the power in each sideband. The term of the sum corresponding to coupling between spin states $\pm \mathcal{I}$ requires a Raman transition to be engineered similarly. For this case, the polarization of the two Raman beams can be chosen to be a superposition of circular and linear polarization in order to allow both the transitions with $\Delta m=1,2$; L. Fallani (private communication).

[44] S. Capponi, P. Lecheminant, and K. Totsuka, Ann. Phys. (Amsterdam) 367, 50 (2016).

[45] O. Boada, A. Celi, J. I. Latorre, and M. Lewenstein, Phys. Rev. Lett. 108, 133001 (2012).

[46] Y. Oreg, E. Sela, and A. Stern, Phys. Rev. B 89, 115402 (2014).
[47] R. B. Laughlin, Phys. Rev. B 23, 5632(R) (1981).

[48] G. Rigolin, G. Ortiz, and V. H. Ponce, Phys. Rev. A 78, 052508 (2008); G. Rigolin and G. Ortiz, Phys. Rev. A 90, 022104 (2014).

[49] See Supplemental Material at http://link.aps.org/ supplemental/10.1103/PhysRevLett.118.230402 for further information on the next-to adiabatic expansion leading to Eqs. (3) and (4), the case of pumping in the synthetic dimension, the exactly-solvable model, and the adiabatic preparation of the magnetic crystal.

[50] M. Mottonen, J. P. Pekola, J. J. Vartiainen, V. Brosco, and F. W. J. Hekking, Phys. Rev. B 73, 214523 (2006).

[51] V. Brosco, R. Fazio, F. W. J. Hekking, and A. Joye, Phys. Rev. Lett. 100, 027002 (2008).

[52] F. Wilczek and A. Zee, Phys. Rev. Lett. 52, 2111 (1984).

[53] V. S. Varadarajan, Perspectives in Geometry and Representation Theory (Hindustan Book Agency, New Delhi, 2003), pp. 502-541.

[54] E. Sela, M. Punk, and M. Garst, Phys. Rev. B 84, 085434 (2011).

[55] E. Cornfeld and E. Sela, Phys. Rev. B 92, 115446 (2015).

[56] J. K. Jain, Composite Fermions (Cambridge University Press, New York, 2007).

[57] Q. Niu, D. J. Thouless, and Y.-S. Wu, Phys. Rev. B 31, 3372 (1985).

[58] U. Schollwöck, Ann. Phys. (Amsterdam) 326, 96 (2011).

[59] P. Marra, R. Citro, and C. Ortix, Phys. Rev. B 91, 125411 (2015).

[60] T. S. Zeng, W. Zhu, and D. N. Sheng, Phys. Rev. B 94, 235139 (2016). 